Infect Control Hosp Epidemiol. 2018 October ; 39(10): 1189-1195. doi:10.1017/ice.2018.184.

\title{
The Projected Burden of Complex Surgical Site Infections following Hip and Knee Arthroplasty among Adults in the United States, 2020 through 2030
}

\author{
Hannah Wolford, MSPH, Kelly Hatfield, MSPH, Prabasaj Paul, PhD, Sarah H. Yi, PhD, and \\ Rachel B. Slayton, PhD \\ Division of Healthcare Quality Promotion, Centers for Disease Control and Prevention, Atlanta, \\ GA, United States
}

\begin{abstract}
Background: As the U.S. population ages, the number of hip and knee arthroplasties is expected to increase. Because surgical site infections (SSIs) following these procedures contribute substantial morbidity, mortality and costs, we projected SSIs expected to occur from 2020 through 2030.
\end{abstract}

Methods: We used a stochastic Poisson process to project the number of primary and revision arthroplasties and SSIs. Primary arthroplasty rates were calculated using annual estimates of hip and knee arthroplasty stratified by age and gender from the 2012-2014 Nationwide Inpatient Sample and standardized by census population data. Revision rates, dependent on time from primary procedure, were obtained from published literature and uniformly applied for all ages and genders. Stratified complex SSI rates for arthroplasties were obtained from 2012-2015 National Healthcare Safety Network data. To evaluate the possible impact of prevention measures, we recalculated the projections with a SSI rate reduced by $30 \%$, the national target established by the U.S. Department of Health and Human Services (HHS).

Results: Without a reduction in SSI rates, we projected an increase in complex SSIs following hip and knee arthroplasty of 14\% between 2020 and 2030. We projected a total burden of 77,654 SSIs, but meeting the $30 \%$ rate reduction could prevent 23,297 of these SSIs.

Conclusions: Given current SSI rates, we project that complex SSI burden for primary and revision arthroplasty may increase due to an aging population. Reducing the SSI rate to the national HHS target could prevent 23,000 SSIs and reduce subsequent morbidity, mortality and Medicare costs.

\section{Introduction}

Hip and knee arthroplasties improve the quality of life for patients who receive elective surgery. ${ }^{1,2}$ The demand for arthroplasty is anticipated to increase due to the aging U.S. population and projected increases in obesity prevalence, leading to a concomitant increase

Correspondence: Hannah Wolford, 1600 Clifton Road MS A-16, Atlanta, GA 30329, 404-798-3490, Lin5@cdc.gov. Preliminary findings were presented in part as an abstract at the IDWeek 2017 Annual Meeting in San Diego, CA. 
in the incidence of SSI. ${ }^{3-10}$ As of 2009, for every 1,000 hip and knee arthroplasties, roughly 6.9 patients developed a complex surgical site infection (SSI). ${ }^{11}$ SSIs are associated with increased perioperative mortality rate, length of stay, cost of in-hospital care and additional procedures to address the infection. ${ }^{12}$ To try to reduce these burdens, the U.S. Department of Health and Human Services (HHS) has established a goal of lowering SSI rates by $30 \%$ by year 2020 in the National Action Plan to Prevent Health Care-Associated Infections: Road Map Elimination (HAI Action Plan). ${ }^{13}$

Previous studies have reported current SSI rates while assessing the infection burden $6,11,14$; however, these rates do not incorporate changes over time in burden and trends of arthroplasties and SSIs. Without considering how changes in population denominators over time will affect the numbers of arthroplasties performed or the risk of SSI, we cannot reliably demonstrate the potential increase in infection and economic burden. Common conditions such as obesity and diabetes have been associated with SSIs and may be changing at the population level. ${ }^{6-10}$ Burden estimates incorporating such information could be used to set healthcare- associated infection (HAI) policy and identify facilities for targeted intervention. ${ }^{15,16}$

To estimate the potential impact of achieving the HHS goal for lowering SSIs while accounting for underlying time trends in population counts and intensity of arthroplasty, this analysis projected primary and revision hip and knee arthroplasty SSIs using estimated and targeted SSI rates from 2020 through 2030 and assessed trends over time. We also assessed the effect of potential trends in comorbidities, by conducting a secondary analysis modelling increasing obesity using BMI-stratified rates.

\section{Methods}

We used a stochastic Poisson process to model the number of primary and revision hip and knee arthroplasties and subsequent SSIs. Surgery and SSI burden was projected annually using 4 separate Poisson models: primary knee, primary hip, revision knee, and revision hip. Arthroplasty rates were applied to the baseline population to create a cohort of individuals who had surgery. The SSI rates were then applied to the resulting cohort. This cycle was repeated annually with the baseline population aging by one year, excluding individuals who died or previously had surgery and including individuals who aged into each cohort (Fig. 1). A second cohort of patients who have previously had an arthroplasty were included in a separate model that applied rates of revision arthroplasties and subsequent SSIs in the same manner. Mathematical computations are described more fully in the Appendix. We selected a static model because (1) the infection rates were low, (2) SSIs have different infection etiologies, and (3) previous studies have shown clustering has little effect on overall infection rates. ${ }^{17}$

Each model was run from January 1, 2020 through December 31, 2030. We used the most recently available data for arthroplasty and SSI rates, as described in the Model Inputs section. Age and gender cohorts were defined as males and females restricted to four age categories: $18-44,45-64,65-84$ and over 85 years old. 
Confidence intervals were calculated for each annual estimate by combining the parameter and Poisson variance. The Poisson variance of each projection was equal to the mean of the projection. Parameter variance was estimated using a bootstrapping method. We assigned a normal distribution to each age- and gender-stratified arthroplasty and SSI rate based on their mean and standard deviation. Next, we ran 1,000 trials of the Poisson process, randomly selecting rates from each normal distribution. We estimated our parameter variance as the variance of these trials.

\section{Model Inputs}

Primary Procedures-For our primary arthroplasty models, we created a synthetic population to match the 2015 U.S. Census Bureau population projections for each birth cohort, stratified by age, sex, race, and Hispanic ethnicity. ${ }^{18}$ Our population of adults increased from $247,733,509$ in 2015 to $268,898,729$ in 2030.

Primary arthroplasty rates were estimated for years 2012 to 2014 for each age and gender cohort from the National Inpatient Sample (NIS). The number of arthroplasties were identified using International Classification of Disease, Ninth Revision, Clinical Modification (ICD-9-CM) procedure codes located in any position on the discharge record. For hip arthroplasty, we used codes for primary total hip replacement (81.51) and primary partial hip replacement (81.52); for knee arthroplasty we used the code for primary knee replacement (81.54). Hip and knee arthroplasties were standardized by resident population estimates (i.e. number of arthroplasties divided by number of residents for each cohort) from the U.S. Census, and then averaged across years 2012 to 2014. These averages were input into the model as time-constant primary procedure rates. We only considered inpatient procedures, as outpatient procedures are rare $(0.75 \%-6.2 \%$ of hip and knee arthroplasties). Some studies have shown no significant difference between adverse events following outpatient vs. inpatient surgery, however outpatient surgical patients tend to have fewer risk factors for surgical complications. ${ }^{19,20}$

The SSI rates were estimated from National Healthcare Safety Network (NHSN) data following primary hip and knee arthroplasty for years 2012 through 2015 stratified by age and gender. Counts of complex SSIs (i.e. deep incisional and organ/space SSIs identified within 90 days of the operative procedure) were standardized by NHSN arthroplasty totals, averaged across years 2012 through 2015, and were input into the model as time-constant infection rates. $^{21}$

For this analysis, to increase precision in the stratified rate estimates, we used constant SSI rates estimated from pooled 2012-2015 NHSN data for each gender and age strata. Overall, our pooled infection rate $(0.56 \%$ for hips and $0.33 \%$ for knees) did not significantly differ from 2015 estimates ( $>0.05$ for both hips and knees). Thus, we used the overall pooled rates in our modeling.

Revision Procedures-Revisions are an infrequent outcome of a primary arthroplasty and can occur at any time following the initial arthroplasty. ${ }^{22}$ We created a cohort of individuals eligible for a revision who had previously had a primary procedure. This cohort 
was estimated from 2000 through 2014 using the NIS, and obtained from our primary arthroplasty model from 2015 onward.

Revision rates were based on published literature sources estimating the risk of revision following primary arthroplasties. Sources showed similar rates of revision for hip and knee arthroplasty (ranging from $81.2 \%-96 \%$ implant survival at 10 years). ${ }^{22-26}$ For this study, we applied rates from Katz, et al. to all age and gender cohorts for all arthroplasties. ${ }^{22} \mathrm{We}$ selected the data from Katz, et al. because their rates were calculated using a large administrative dataset (vs a single facility) over a 12 year time period.

Revision SSI rates were estimated from NHSN data following revision hip and knee arthroplasty for years 2012 through 2015 stratified by age and gender. We utilized SSI counts standardized by NHSN arthroplasty totals, averaged across years 2012 to 2015 , and input into the model as time-constant infection rates.

Mortality Rate-Background mortality was obtained from the U.S. Census Bureau estimates and projections for 2000 through 2030 stratified by year of age (until age 85 where the mortality rate applied to age 85 and over). The model applied the mortality rate to individuals in the simulated baseline population who had not already received an arthroplasty (primary or revision dependent on model) based on the current year and current age of the individual.

HHS Action Plan Goals-To assess the potential impact the HHS target rate reduction would have on SSI burden from 2020 to 2030, we reduced each stratified rate in our model by $30 \%$ beginning in 2020 and sustained through 2030 . We compared these results with the original projections.

Obesity Rate-To assess the model's sensitivity to changes in comorbidities, we conducted a secondary analysis utilizing projected obesity increases and stratified obesity rates. Finkelstein, et al. projected that obesity (defined as BMI 230) would increase from $34 \%$ in 2015 to $42 \%$ in $2030 .{ }^{27}$ Using these projections, we stratified the baseline population into non-obese and obese. We obtained stratified relative risks for obese and non-obese populations for primary arthroplasties using 2012-2014 Cerner HealthFacts, a large electronic health records database. We estimated BMI-stratified SSI rates from 2012-2015 NHSN data. We applied these relative risks as multipliers to our previous primary arthroplasty and SSI rates to calculate the estimated obese and non-obese arthroplasty and SSI rates. The result was a baseline population, arthroplasty, and SSI rate for each gender, age and BMI cohort (Appendix Table 1). We ran the primary model with the new cohorts with and without increasing population obesity rates from 2015 through 2030.

\section{Results}

Our model projected 15,820,475 primary and revision hip and knee arthroplasties and 77,653 subsequent SSIs from 2020 through 2030. The number of arthroplasties increased $13 \%$, with a $14 \%$ increase in total SSIs, representing 179,106 additional arthroplasties and 937 additional SSIs in 2030 compared to 2020. (Figure 2, Appendix Table 2) 
Primary and revision hip arthroplasties combined were projected to increase by $15 \%$ and subsequent SSIs increased by $16 \%$ to 4,063 infections in 2030 (Figure 2, Appendix Table 2). The model projected primary and revision knee arthroplasties would increase by $12 \%$ and subsequent SSIs following knee arthroplasties would increase by 13\%, with 3,443 SSIs in 2030 (Figure 2, Appendix Table 2). Furthermore, SSIs following hip arthroplasties contributed 54\% of the total SSIs following arthroplasties.

Arthroplasty revisions accounted for $10 \%$ of all arthroplasties $(1,512,661$ cumulative revisions across all years). From 2020 through 2030, the model projected revisions would increase 23\% (Figure 2, Appendix Table 2). SSIs following revision increased by $21 \%$ and accounted for $26 \%$ of all SSIs (Figure 2, Appendix Table 2).

Most arthroplasties and SSIs were performed on patients aged between 65 and 84 years. The percent of the total arthroplasties and SSIs increased between 2020 and 2030, from 52\% and 59\% of total arthroplasties and SSIs respectively in 2020 to 59\% and 65\% in 2030 (Figure 3). (Table 1)

Assuming the HHS targeted goal of a 30\% reduction in SSI rates is achieved, our model projected an average total of 54,356 SSIs (Table 2), or 23,297 fewer SSIs over 11 years.

An increased obesity rate contributed 796,840 more arthroplasties from 2020 through 2030 (Table 3) than if the obesity rate remained stable. Similarly, 5,570 additional SSIs (Table 3) were projected.

\section{Discussion \\ Summary of Results}

The results of our study provide overall arthroplasty and SSI burden projections for the years 2020 through 2030, assuming constant rates. We project a 13\% increase in arthroplasties and $14 \%$ increase in SSIs, with 60-70\% of arthroplasties and SSIs occurring in the 65 and older age group. Our model demonstrates that even with stable arthroplasty and SSI rates, demographic changes in the U.S. population will result in more arthroplasties and SSIs, as well as an increased public health burden. The HHS previously set a target for a $25 \%$ reduction from 2009 SSI rates, and only achieved an $18 \%$ reduction by $2014 .{ }^{13}$ In an update to the HAI Action Plan, the HHS defined a new target of a 30\% reduction from 2015 SSI rates. This study shows achieving this goal would prevent 23,000 SSIs, demonstrating the importance of prevention in reducing the health and subsequent economic impact of SSIs.

\section{Public Health Impact}

Our study predicts burden for attributable SSIs following hip and knee arthroplasties (including only the first arthroplasty revision). While our burden estimates for procedures and infections may represent a subset of other estimates ${ }^{28}$ because we selected only complex SSIs as defined by $\mathrm{NHSN}^{21}$ following hip and knee arthroplasty, we feel these infections are more influential as they represent more severe and costly infections occurring in common procedures. It is important to understand the burden of this subset of infections, because 
NHSN infection rates are used by HHS to track progress on eliminating HAIs, and represent areas of measured improvement.

When developing HAI interventions, policy makers set health priorities for prevention. The CDC recommends that states identify priority infections when developing their policy interventions. ${ }^{16}$ Estimates of future burden may help prioritize the development of new and improved prevention strategies. Identifying facilities with a higher burden of SSIs will become increasingly important in future years as burden increases. Comprehensive guidelines on strategies to reduce SSIs were recently updated with specific recommendations for infections following prosthetic joint arthroplasties that have the potential to reduce rates of these infections. ${ }^{29}$ Investment in these recommendations may decrease patient morbidity and save substantial hospital costs.

However, SSIs are relatively rare in comparison to other HAIs. Using national estimates derived from the 2011 multistate point-prevalence survey for central line-associated bloodstream infections (CLABSIs), we would expect 171,600 total CLABSIs across 11 years versus roughly 78,000 SSIs following hip and knee arthroplasty procedures alone. ${ }^{30}$ However, SSIs are a significant component of the overall burden of HAI costs, and account for anywhere from 3 to 22 times the patient hospital costs of more common conditions such as CLABSIs, ventilator-associated pneumonia, catheter-associated urinary tract infections and Clostridium difficile infections. ${ }^{31}$ Additionally, SSIs continue to accrue costs long after the initial diagnosis and treatment. Yi et al. ${ }^{14}$ found that patients who had SSI following hip and knee arthroplasty procedures required $\$ 53,470$ in excess Medicare reimbursement in the four years following a procedure, in comparison to those who did not experience an SSI.

Medicare will be responsible for much of the costs associated with SSIs following hip and knee arthroplasty. In 2014, Medicare paid for 55\% of knee arthroplasties and 59\% of hip arthroplasties claims nationally ${ }^{32}$, and our model shows that the majority of SSIs from 2020 through 2030 following arthroplasties would occur in a Medicare-eligible population (patients age 65 and older). Multiplying the likely proportion of SSIs paid for by Medicare by Yi et al's excess Medicare reimbursement, we found Medicare would likely be responsible for $>\$ 2.4$ billion in reimbursements attributable to SSIs from 2020 through 2030. Although we do not have an estimate for private payer reimbursement, extrapolating the Medicare reimbursement amount to the total population translates to $\$ 4.2$ billion in reimbursement (by both private and Medicare insurance) attributable to SSIs following hip and knee arthroplasty.

\section{Rate Reduction Impact}

We project achieving the HHS goal of a $30 \%$ reduction in the rate of SSIs would prevent > 20,000 SSIs following hip and knee arthroplasty alone and may have substantial impact on subsequent patient morbidity and hospital costs. Reaching the goal could result in $\$ 712$ million savings in Medicare reimbursement (assuming \$53,470 in Medicare reimbursement per SSI). ${ }^{14}$ Notably, these infections represent only a small portion of the total number of SSIs. Extrapolating that cost number to the total population could result in a reduction of $\$ 1.2$ billion in total reimbursement. 
Our analysis has several limitations that should be considered. Our study assumed stable rates obtained from historical data and published literature for arthroplasties and SSIs, stratified by age, gender, and obesity status. Our infection rates relied on self-reported surveillance data from NHSN. We did not account for rate changes due to outside factors suggested by other studies including increasing demand for the surgery or comorbidities, such as diabetes, depression or cardiac arrhythmia, ${ }^{5-10}$ which we would expect to lead to increases in arthroplasties and SSIs (similar to our obesity analysis). Additionally, our arthroplasty rates did not include outpatient or same-day discharge surgeries $(0.75-6.2 \%$ of hip and knee arthroplasties) which are infrequent but may potentially increase in the future. Thus, our projections may have been underestimated. ${ }^{19,33}$ Due to data limitations, we were unable to directly track patients across time or to obtain revision rates; thus, we estimated a revision rate from Medicare claims data for all procedures, regardless of payer. ${ }^{22}$ Also, data regarding SSIs resulting from a revision arthroplasty can also be difficult to obtain. NHSN definitions state that if several procedures are performed on different dates prior to an infection, the SSI is attributed to the most recent procedure prior to the infection date. Additionally, if evidence of an infection is present at the time of the procedure, an SSI will be attributed to that procedure. ${ }^{21}$

Despite low and constant rates, this analysis projected an increased SSI burden in the years 2020 through 2030 from hip and knee arthroplasty. Subsequent hospital and Medicare costs may increase by millions of dollars due to SSIs following arthroplasty and the growing population over the age of 65 . Achieving SSI prevention goals may have a substantial impact on reducing HAI burden.

\section{Supplementary Material}

Refer to Web version on PubMed Central for supplementary material.

\section{Acknowledgements}

The authors do not report any financial support or conflicts of interest.

\section{References}

1. Lovald ST, Ong KL, Lau EC, Schmier JK, Bozic KJ, Kurtz SM. Mortality, cost, and downstream disease of total hip arthroplasty patients in the medicare population. J Arthroplasty 2014;29:242246. [PubMed: 23711799]

2. Lovald ST, Ong KL, Lau EC, Schmier JK, Bozic KJ, Kurtz SM. Mortality, cost, and health outcomes of total knee arthroplasty in Medicare patients. J Arthroplasty 2013;28:449-454. [PubMed: 23142446]

3. Kurtz SM, Ong KL, Lau E, Bozic KJ. Impact of the economic downturn on total joint replacement demand in the United States: updated projections to 2021. J Bone Joint Surg Am 2014;96:624-630. [PubMed: 24740658]

4. Kurtz SM, Ong KL, Schmier J, et al. Future clinical and economic impact of revision total hip and knee arthroplasty. J Bone Joint Surg Am 2007;89 Suppl 3:144-151. [PubMed: 17908880]

5. Pollock M, Somerville L, Firth A, Lanting B. Outpatient total hip arthroplasty, total knee arthroplasty, and unicompartmental knee arthroplasty: a systematic review of the literature. JBJS Rev 2016;4. 
6. Bozic KJ, Lau E, Kurtz S, et al. Patient-related risk factors for periprosthetic joint infection and postoperative mortality following total hip arthroplasty in Medicare patients. J Bone Joint Surg Am 2012;94:794-800. [PubMed: 22552668]

7. Bozic KJ, Ward DT, Lau EC, et al. Risk factors for periprosthetic joint infection following primary total hip arthroplasty: a case control study. J Arthroplasty 2014;29:154-156. [PubMed: 23702271]

8. Lee QJ, Mak WP, Wong YC. Risk factors for periprosthetic joint infection in total knee arthroplasty. J Orthop Surg (Hong Kong) 2015;23:282-286. [PubMed: 26715700]

9. Peter WF, Dekker J, Tilbury C, et al. The association between comorbidities and pain, physical function and quality of life following hip and knee arthroplasty. Rheumatol Int 2015;35:1233-1241. [PubMed: 25586654]

10. Singh JA, Vessely MB, Harmsen WS, et al. A population-based study of trends in the use of total hip and total knee arthroplasty, 1969-2008. Mayo Clin Proc 2010;85:898-904. [PubMed: 20823375]

11. Berrios-Torres SI, Yi SH, Bratzler DW, et al. Activity of commonly used antimicrobial prophylaxis regimens against pathogens causing coronary artery bypass graft and arthroplasty surgical site infections in the United States, 2006-2009. Infect Control Hosp Epidemiol 2014;35:231-239. [PubMed: 24521586]

12. Poultsides LA, Ma Y, Della Valle AG, Chiu YL, Sculco TP, Memtsoudis SG. In-hospital surgical site infections after primary hip and knee arthroplasty--incidence and risk factors. J Arthroplasty 2013;28:385-389. [PubMed: 23142444]

13. National targets and metrics. 2017 https://health.gov/hcq/prevent-hai-measures.asp Accessed October 12, 2017.

14. Yi SH, Baggs J, Culler SD, Berrios-Torres SI, Jernigan JA. Medicare reimbursement attributable to periprosthetic joint infection following primary hip and knee arthroplasty. J Arthroplasty 2015;30:931-938 e932. [PubMed: 25727999]

15. Soe MM, Gould CV, Pollock D, Edwards J. Targeted assessment for prevention of healthcareassociated infections: a new prioritization metric. Infect Control Hosp Epidemiol 2015;36:1379_ 1384. [PubMed: 26310913]

16. Policies for Eliminating Healthcare-Associated Infections: Lessons learned from state stakeholder engagement. The Association of State and Territorial Health Officials and the Centers for Disease Control;2012.

17. Baker AW, Dicks KV, Durkin MJ, et al. Epidemiology of surgical site infection in a community hospital network. Infect Control Hosp Epidemiol 2016;37:519-526. [PubMed: 26864617]

18. 2014 National Population Projections U.S. Census; 2014.

19. Sher A, Keswani A, Yao DH, Anderson M, Koenig K, Moucha CS. Predictors of Same-Day Discharge in Primary Total Joint Arthroplasty Patients and Risk Factors for Post-Discharge Complications. J Arthroplasty 2016.

20. Aynardi M, Post Z, Ong A, Orozco F, Sukin DC. Outpatient surgery as a means of cost reduction in total hip arthroplasty: a case-control study. HSS J 2014;10:252-255. [PubMed: 25264442]

21. Procedure-associated module SSI.: National Healthcare Safety Network; 2017: https:// www.cdc.gov/nhsn/pdfs/pscmanual/9pscssicurrent.pdf.

22. Katz JN, Wright EA, Wright J, et al. Twelve-year risk of revision after primary total hip replacement in the U.S. Medicare population. J Bone Joint Surg Am 2012;94:1825-1832. [PubMed: 23079874]

23. Duffy GP, Crowder AR, Trousdale RR, Berry DJ. Cemented total knee arthroplasty using a modern prosthesis in young patients with osteoarthritis. J Arthroplasty 2007;22:67-70. [PubMed: 17823019]

24. Guo L, Yang L, Briard JL, Duan XJ, Wang FY. Long-term survival analysis of posterior cruciateretaining total knee arthroplasty. Knee Surg Sports Traumatol Arthrosc 2012;20:1760-1765. [PubMed: 22072324]

25. Kearns SR, Jamal B, Rorabeck CH, Bourne RB. Factors affecting survival of uncemented total hip arthroplasty in patients 50 years or younger. Clin Orthop Relat Res 2006;453:103-109. [PubMed: 17006361] 
26. Ong KL, Lau E, Suggs J, Kurtz SM, Manley MT. Risk of subsequent revision after primary and revision total joint arthroplasty. Clin Orthop Relat Res 2010;468:3070-3076. [PubMed: 20499292]

27. Finkelstein EA, Khavjou OA, Thompson H, et al. Obesity and severe obesity forecasts through 2030. Am J Prev Med 2012;42:563-570. [PubMed: 22608371]

28. Kurtz SM, Lau E, Watson H, Schmier JK, Parvizi J. Economic burden of periprosthetic joint infection in the United States. J Arthroplasty 2012;27:61-65 e61. [PubMed: 22554729]

29. Berrios-Torres SI, Umscheid CA, Bratzler DW, et al. Centers for disease control and prevention guideline for the prevention of surgical site infection, 2017. JAMA Surg 2017;152:784-791. [PubMed: 28467526]

30. Magill SS, Edwards JR, Bamberg W, et al. Multistate point-prevalence survey of health careassociated infections. N Engl J Med 2014;370:1198-1208. [PubMed: 24670166]

31. Scott RD. The Direct Medical Costs of healthcare-Associated Infections in U.S. Hospitals and the Benefits of Prevention. In: Promotion DoHQ, ed: Centers for Disease Control and Prevention; 2009.

32. National inpatient sample. In: Project $\mathrm{HCaU}$, ed2016.

33. Bovonratwet P, Webb ML, Ondeck NT, et al. Definitional Differences of 'Outpatient' Versus 'Inpatient' THA and TKA Can Affect Study Outcomes. Clin Orthop Relat Res 2017. 


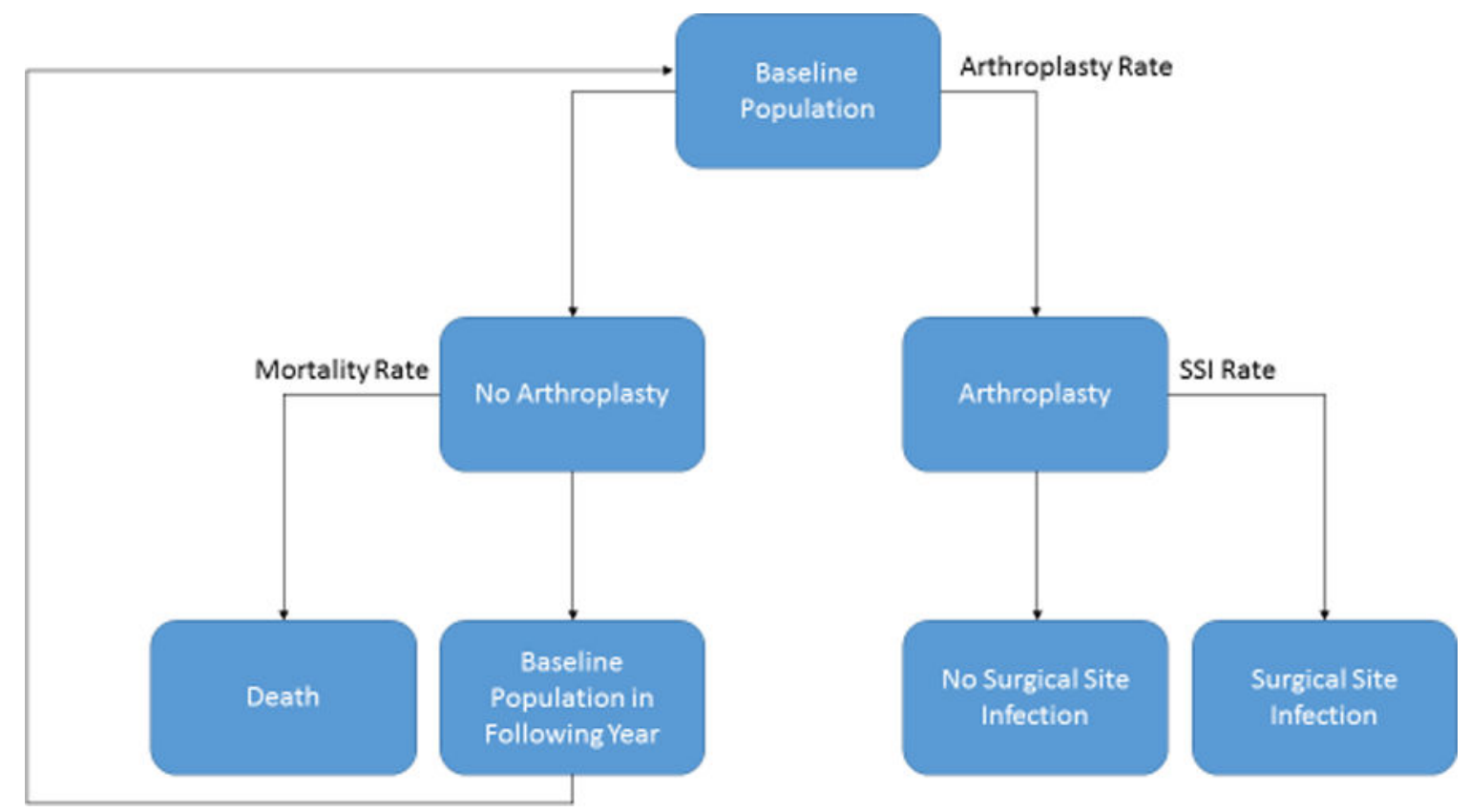

Figure 1.

Flow chart depicting general model for obtaining annual arthroplasty and surgical site infection (SSI) counts. Arthroplasty rates were applied to the baseline population, and SSI rates are applied to this resulting population receiving arthroplasties. Mortality rates were not applied to individuals between arthroplasty and SSI branches (by definition 90 days or less). The cycle was repeated annually, with the baseline population as the population aged 1 year from the previous year without an arthroplasty or death. The model was run separately for primary and revision hip and knee arthroplasty (4 separate models). 
Projected Hip Arthroplasty SSIs

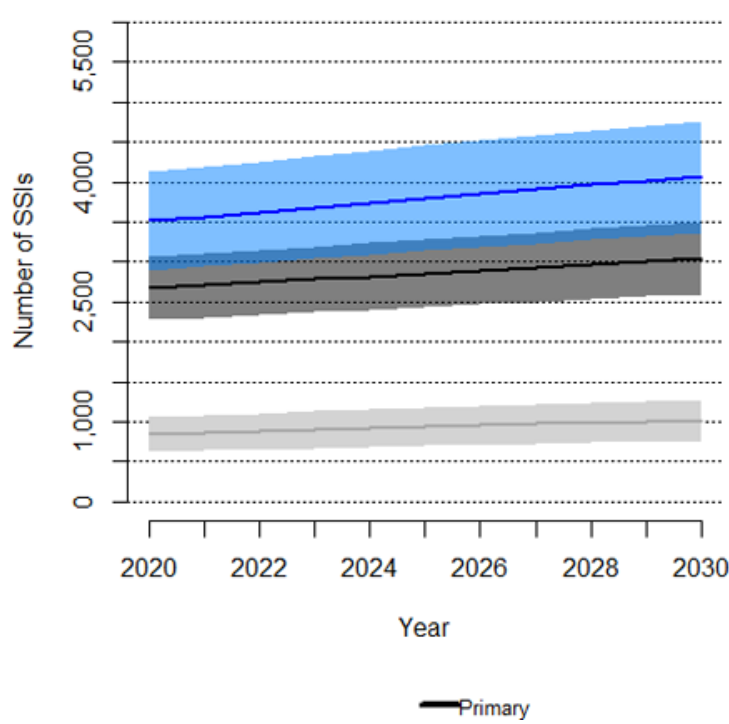

Projected Knee Arthroplasty SSIs

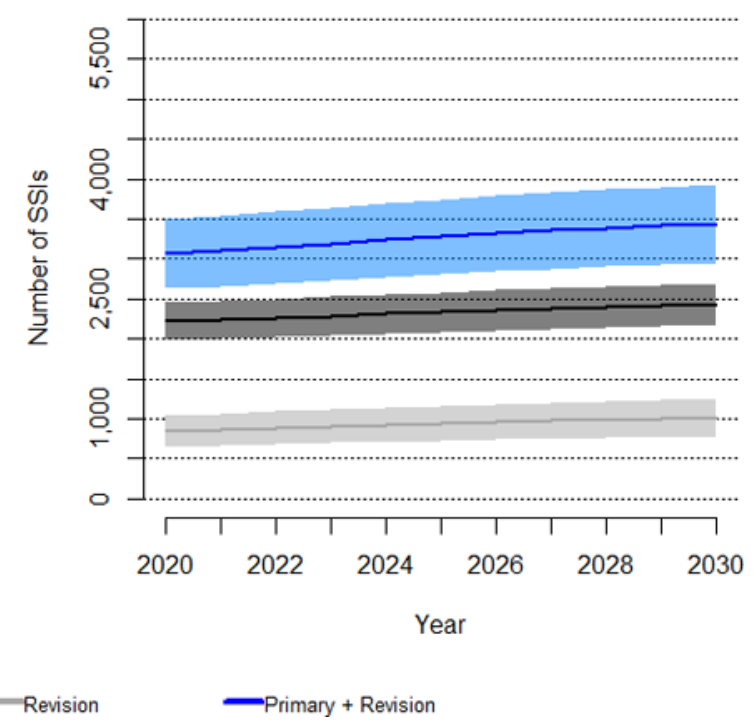

Figure 2.

Projected 2020 to 2030 complex surgical site infections (SSIs) following primary and revision hip and knee arthroplasty with $95 \%$ confidence intervals. 
2020

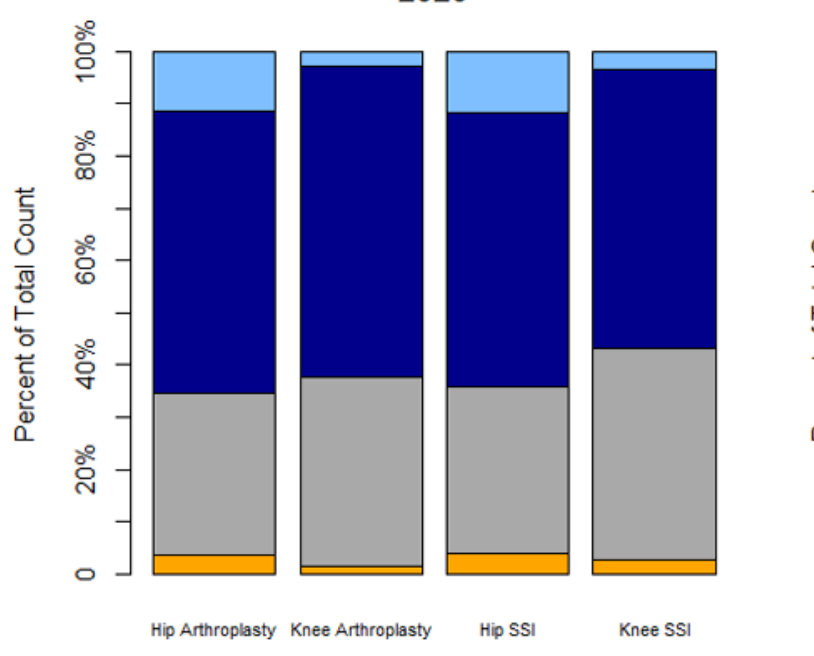

Age 18.44

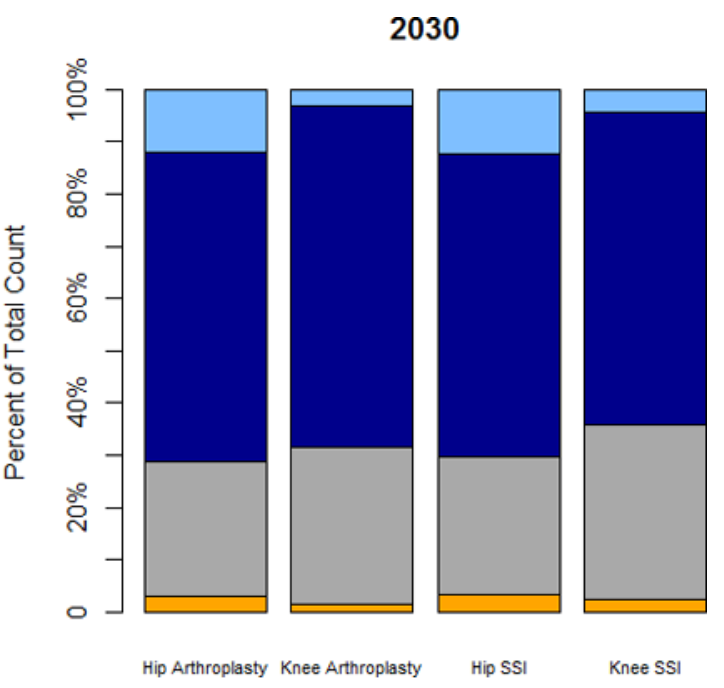

Figure 3.

Comparison of the percent of projected hip and knee arthroplasties and surgical site infections (SSIs) in each age cohort between 2020 and 2030. 
Table 1.

Primary and revision model inputs for hip and knee arthroplasty

\begin{tabular}{|l|l|l|l|l|l|}
\hline Age Cohort & Gender & Primary Arthroplasty Rate & Primary SSI Rate $^{* *}$ & Revision Arthroplasty Rate $^{*}$ & Revision SSI Rate $^{* *}$ \\
\hline Hip Arthroplasty & \multicolumn{5}{|l|}{} \\
\hline $18-44$ & Male & $0.02 \%$ & $0.52 \%$ & $2 \% / 1.5 \% / 1 \%$ & $2.03 \%$ \\
\hline $45-64$ & Male & $0.19 \%$ & $0.54 \%$ & $2 \% / 1.5 \% / 1 \%$ & $2.05 \%$ \\
\hline $65-85$ & Male & $0.46 \%$ & $0.58 \%$ & $2 \% / 1.5 \% / 1 \%$ & $1.79 \%$ \\
\hline $85+$ & Male & $0.76 \%$ & $0.63 \%$ & $2 \% / 1.5 \% / 1 \%$ & $1.85 \%$ \\
\hline $18-44$ & Female & $0.01 \%$ & $0.61 \%$ & $2 \% / 1.5 \% / 1 \%$ & $1.83 \%$ \\
\hline $45-64$ & Female & $0.18 \%$ & $0.56 \%$ & $2 \% / 1.5 \% / 1 \%$ & $2.05 \%$ \\
\hline $65-85$ & Female & $0.64 \%$ & $0.50 \%$ & $2 \% / 1.5 \% / 1 \%$ & $1.63 \%$ \\
\hline $85+$ & Female & $1.02 \%$ & $0.56 \%$ & $2 \% / 1.5 \% / 1 \%$ & $1.42 \%$ \\
\hline Knee Arthroplasty & & & & $2 \%$ \\
\hline $18-44$ & Male & $0.01 \%$ & $0.66 \%$ & $2 \% / 1.5 \% / 1 \%$ & $1.55 \%$ \\
\hline $45-64$ & Male & $0.26 \%$ & $0.44 \%$ & $2 \% / 1.5 \% / 1 \%$ & $1.28 \%$ \\
\hline $65-85$ & Male & $0.76 \%$ & $0.35 \%$ & $2 \% / 1.5 \% / 1 \%$ & $0.88 \%$ \\
\hline $85+$ & Male & $0.28 \%$ & $0.45 \%$ & $2 \% / 1.5 \% / 1 \%$ & $0.72 \%$ \\
\hline $18-44$ & Female & $0.01 \%$ & $0.37 \%$ & $2 \% / 1.5 \% / 1 \%$ & $1.12 \%$ \\
\hline $45-64$ & Female & $0.39 \%$ & $0.29 \%$ & $2 \% / 1.5 \% / 1 \%$ & $0.92 \%$ \\
\hline $65-85$ & Female & $1.07 \%$ & $0.22 \%$ & $2 \% / 1.5 \% / 1 \%$ & $0.84 \%$ \\
\hline $85+$ & Female & $0.26 \%$ & $0.22 \%$ & $2 \% / 1.5 \% / 1 \%$ & \\
\hline
\end{tabular}

* Primary arthroplasty rates were obtained using the 2012-2014 National Inpatient Sample

** Primary and revision SSI rates were obtained from the 2012-2015 National Healthcare Safety Network ****

Revision arthroplasty rates were obtained from Katz et al. ${ }^{22}$ Rates were dependent on the time following a primary arthroplasty so we applied different rates to patients one year following an arthroplasty, two years following an arthroplasty, and then all subsequent years following an arthroplasty 
Table 2.

Comparison of projected 2020 to 2030 primary and revision hip and knee arthroplasty surgical site infections (SSIs) with and without an infection rate reduction of $30 \%$.

\begin{tabular}{|c|c|c|c|c|c|c|c|c|c|c|c|c|}
\hline & 2020 & 2021 & 2022 & 2023 & 2024 & 2025 & 2026 & 2027 & 2028 & 2029 & 2030 & Total \\
\hline \multicolumn{13}{|l|}{ All Arthroplasties } \\
\hline SSIs without rate reduction & 6,569 & 6,671 & 6,773 & 6,874 & 6,972 & 7,076 & 7,171 & 7,262 & 7,349 & 7,430 & 7,506 & 77,654 \\
\hline SSIs with rate reduction & 4,598 & 4,670 & 4,741 & 4,812 & 4,881 & 4,953 & 5,020 & 5,083 & 5,144 & 5,200 & 5,254 & 54,356 \\
\hline $\begin{array}{l}\text { SSIs prevented with rate } \\
\text { reduction }\end{array}$ & 1,971 & 2,001 & 2,032 & 2,062 & 2,091 & 2,123 & 2,151 & 2,179 & 2,205 & 2,230 & 2,252 & 23,297 \\
\hline \multicolumn{13}{|c|}{ Primary and Revision Arthroplasties } \\
\hline $\begin{array}{l}\text { Hip SSIs without rate } \\
\text { reduction }\end{array}$ & 3,510 & 3,567 & 3,624 & 3,682 & 3,738 & 3,798 & 3,854 & 3,909 & 3,964 & 4,015 & 4,063 & 41,724 \\
\hline Hip SSIs with rate reduction & 2,457 & 2,497 & 2,537 & 2,577 & 2,617 & 2,658 & 2,698 & 2,736 & 2,775 & 2,810 & 2,844 & 29,206 \\
\hline $\begin{array}{l}\text { SSIs prevented with rate } \\
\text { reduction }\end{array}$ & 1,053 & 1,070 & 1,087 & 1,105 & 1,121 & 1,140 & 1,156 & 1,173 & 1,189 & 1,205 & 1,219 & 12,518 \\
\hline $\begin{array}{l}\text { Knee SSIs without rate } \\
\text { reduction }\end{array}$ & 3,059 & 3,104 & 3,149 & 3,192 & 3,234 & 3,278 & 3,317 & 3,353 & 3,385 & 3,415 & 3,443 & 35,929 \\
\hline Knee SSIs with rate reduction & 2,141 & 2,173 & 2,204 & 2,235 & 2,264 & 2,295 & 2,322 & 2,347 & 2,369 & 2,390 & 2,410 & 25,150 \\
\hline $\begin{array}{l}\text { SSIs prevented with rate } \\
\text { reduction }\end{array}$ & 918 & 931 & 945 & 957 & 970 & 983 & 995 & 1,006 & 1,016 & 1,025 & 1,033 & 10,779 \\
\hline \multicolumn{13}{|l|}{ Primary Arthroplasties } \\
\hline $\begin{array}{l}\text { Hip SSIs without rate } \\
\text { reduction }\end{array}$ & 2,674 & 2,708 & 2,745 & 2,782 & 2,818 & 2,859 & 2,898 & 2,936 & 2,976 & 3,011 & 3,046 & 31,453 \\
\hline Hip SSIs with rate reduction & 1,872 & 1,896 & 1,921 & 1,947 & 1,973 & 2,002 & 2,029 & 2,055 & 2,083 & 2,108 & 2,132 & 22,018 \\
\hline $\begin{array}{l}\text { SSIs prevented with rate } \\
\text { reduction }\end{array}$ & 802 & 812 & 824 & 835 & 845 & 857 & 869 & 881 & 893 & 903 & 914 & 9,435 \\
\hline $\begin{array}{l}\text { Knee SSIs without rate } \\
\text { reduction }\end{array}$ & 2,216 & 2,238 & 2,263 & 2,286 & 2,309 & 2,335 & 2,358 & 2,379 & 2,397 & 2,414 & 2,430 & 25,625 \\
\hline Knee SSIs with rate reduction & 1,551 & 1,567 & 1,584 & 1,600 & 1,616 & 1,635 & 1,651 & 1,665 & 1,678 & 1,690 & 1,701 & 17,938 \\
\hline $\begin{array}{l}\text { SSIs prevented with rate } \\
\text { reduction }\end{array}$ & 665 & 671 & 679 & 686 & 693 & 700 & 707 & 714 & 719 & 724 & 729 & 7,687 \\
\hline \multicolumn{13}{|l|}{ Revision Arthroplasties } \\
\hline $\begin{array}{l}\text { Hip SSIs without rate } \\
\text { reduction }\end{array}$ & 836 & 859 & 880 & 900 & 920 & 938 & 956 & 973 & 989 & 1,003 & 1,017 & 10,271 \\
\hline Hip SSIs with rate reduction & 585 & 601 & 616 & 630 & 644 & 657 & 669 & 681 & 692 & 702 & 712 & 7,189 \\
\hline $\begin{array}{l}\text { SSIs prevented with rate } \\
\text { reduction }\end{array}$ & 251 & 258 & 264 & 270 & 276 & 281 & 287 & 292 & 297 & 301 & 305 & 3,082 \\
\hline $\begin{array}{l}\text { Knee SSIs without rate } \\
\text { reduction }\end{array}$ & 843 & 865 & 887 & 906 & 925 & 943 & 959 & 974 & 988 & 1,001 & 1,012 & 10,303 \\
\hline Knee SSIs with rate reduction & 590 & 606 & 621 & 635 & 648 & 660 & 671 & 682 & 691 & 700 & 708 & 7,212 \\
\hline $\begin{array}{l}\text { SSIs prevented with rate } \\
\text { reduction }\end{array}$ & 253 & 259 & 266 & 271 & 277 & 283 & 288 & 292 & 297 & 301 & 304 & 3,091 \\
\hline
\end{tabular}

Infect Control Hosp Epidemiol. Author manuscript; available in PMC 2019 October 01. 
Table 3.

Comparison of projected 2020 to 2030 primary and revision hip and knee arthroplasties and surgical site infections (SSIs) with and without an increasing rate of obesity in the baseline population

\begin{tabular}{|c|c|c|c|c|c|c|c|c|c|c|c|c|}
\hline & 2020 & 2021 & 2022 & 2023 & 2024 & 2025 & 2026 & 2027 & 2028 & 2029 & 2030 & Total \\
\hline \multicolumn{13}{|l|}{ Hip Arthroplasties } \\
\hline Increasing obesity rate & 492,153 & 498,827 & 505,966 & 513,163 & 520,269 & 528,167 & 535,599 & 542,853 & 550,251 & 556,946 & 563,437 & $5,807,631$ \\
\hline Stable obesity rate & 490,818 & 497,235 & 504,119 & 511,066 & 517,925 & 525,576 & 532,767 & 539,785 & 546,954 & 553,422 & 559,682 & $5,779,349$ \\
\hline Difference & 1,335 & 1,592 & 1,847 & 2,097 & 2,344 & 2,591 & 2,832 & 3,068 & 3,297 & 3,524 & 3,755 & 28,282 \\
\hline \multicolumn{13}{|l|}{ Hip SSIs } \\
\hline Increasing obesity rate & 2,693 & 2,748 & 2,805 & 2,862 & 2,919 & 2,980 & 3,039 & 3,097 & 3,157 & 3,212 & 3,266 & 32,778 \\
\hline Stable obesity rate & 2,583 & 2,616 & 2,651 & 2,687 & 2,722 & 2,761 & 2,799 & 2,836 & 2,874 & 2,908 & 2,941 & 30,378 \\
\hline Difference & 110 & 132 & 154 & 175 & 197 & 219 & 240 & 261 & 283 & 304 & 325 & 2,400 \\
\hline \multicolumn{13}{|l|}{ Knee Arthroplasties } \\
\hline Increasing obesity rate & 674,187 & 688,618 & 703,574 & 718,031 & 732,332 & 747,413 & 761,366 & 774,253 & 785,882 & 797,411 & 808,183 & $8,191,250$ \\
\hline Stable obesity rate & 637,982 & 645,427 & 653,436 & 661,052 & 668,595 & 676,913 & 684,270 & 690,744 & 696,182 & 701,618 & 706,473 & $7,422,692$ \\
\hline Difference & 36,205 & 43,191 & 50,138 & 56,979 & 63,737 & 70,500 & 77,096 & 83,509 & 89,700 & 95,793 & 101,710 & 768,558 \\
\hline \multicolumn{13}{|l|}{ Knee SSIs } \\
\hline Increasing obesity rate & 2,059 & 2,105 & 2,153 & 2,199 & 2,245 & 2,294 & 2,339 & 2,382 & 2,421 & 2,460 & 2,497 & 25,154 \\
\hline Stable obesity rate & 1,909 & 1,926 & 1,946 & 1,964 & 1,982 & 2,003 & 2,021 & 2,038 & 2,052 & 2,065 & 2,078 & 21,984 \\
\hline Difference & 150 & 179 & 207 & 235 & 263 & 291 & 318 & 344 & 369 & 395 & 419 & 3,170 \\
\hline
\end{tabular}

